\title{
Poster Abstract: Estimating Behind-the-meter Solar Generation with Existing Measurement Infrastructure
}

\author{
Emre Can Kara \\ SLAC National Accelerator \\ Laboratory \\ Menlo Park, CA 94025 \\ emrecan@slac.stanford.edu \\ Michaelangelo Tabone \\ Stanford University \\ Stanford, CA \\ mtabone@stanford.edu
}

\author{
Ciaran Roberts \\ Lawrence Berkeley National \\ Laboratory \\ Berkeley, CA 94720 \\ cmroberts@lbl.gov
}

\author{
Sila Kiliccote \\ SLAC National Accelerator \\ Laboratory \\ Menlo Park, CA 94025 \\ silak@slac.stanford.edu
}

\author{
Emma M. Stewart \\ Lawrence Berkeley National \\ Laboratory \\ Berkeley, CA 94720 \\ estewart@lbl.gov
}

\begin{abstract}
Real-time PV generation information is crucial for distribution system operations, in particular for switching operations, state-estimation, and management of the voltage at the point of provision. However, most behind-the-meter solar installations are not monitored. Typically, the only information available to the distribution system operator is the installed capacity. Our main purpose in this study is to estimate behind-the-meter PV generation using data from smart meters.
\end{abstract}

\section{INTRODUCTION}

The distribution system is facing a fundamental shift. Behind-the-meter distributed energy resources (DERs) may have significant implications for real-time operations of the distribution system. But often, distribution system operators do not have the necessary visibility into DER availability, flexibility or consumption/generation patterns.

The location of loads and generation within a distribution network plays a crucial role 1. The effect of PV generation on feeder operations vary widely, and can be either positive or negative depending on feeder topology, climate, and the timing of other connected loads 2, 3. Furthermore, 3 shows that using similar profiles for PV generation at different nodes overestimates voltage control actions.

The current state-of-the-art for modeling PV generation are (i) model-based approaches based on PV array geometry and irradiance measurements [4], and (ii) direct measurement of the real-time generation. Both methods have drawbacks. Monitoring each PV system is expensive and may not capture consumers who installed solar without proper permitting. Modeling PV, on the other hand, requires accu-

This manuscript has been authored by SLAC National Accelerator Laboratory and Lawrence Berkeley National Laboratory under Contract Numbers DE-AC0276 SF00515 and DE-AC02-05CH11231 with the U.S. Department of Energy, respectively.

BuildSys '16 November 16-17, 2016, Palo Alto, CA, USA

(C) 2016 Copyright held by the owner/author(s)

ACM ISBN 978-1-4503-4264-3/16/11.

DOI: http://dx.doi.org/10.1145/2993422.2996419

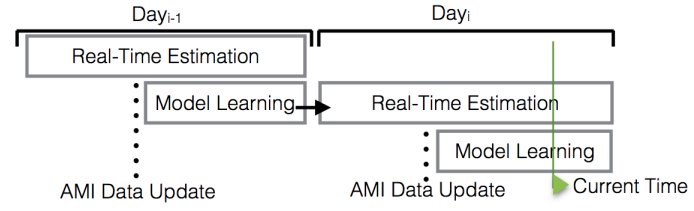

Figure 1: Data updates and estimation framework

rate knowledge of where $\mathrm{PV}$ systems are in the system, and their geometries. Furthermore, research suggests that these models can be inaccurate 5 .

In this paper, we present a proof-of-concept method for estimating the PV generation at each household on a distribution feeder using only AMI data. This method provides visibility into where PV are located in the distribution system, the rated capacity of PV systems, as well as their production. We envision this work contributing toward a new framework for monitoring and control of DER in distributions systems, shown in Figure 1. In this framework, models will train on spatially rich data that is obtained with delaysuch as AMI data. These models are designed so that they can be applied to data that are available in real-time but aggregated, such as those from supervisory control and data acquisition systems (SCADA) and weather stations.

\section{METHOD}

Previously, we developed a methodology to estimate PV generation at the feeder level using measurements from microsynchrophasors 6]. In this paper, we expand this methodology by disaggregating PV generation at the individual household level by adding the AMI data information as a constraint. Furthermore, since the reactive power measurements are unavailable, we are using hourly temperature data to model aggregate loads at the distribution feeder level.

We suggest the following learning and estimation strategy. At any given point in time $t$, we define the net load at the substation as $N L_{t}$, the aggregate load as $A L_{t}$, and individual solar generation $S_{t}^{i}$ for each household $i$. We also define an irradiance proxy measurement as $\phi_{t}{ }^{1}$ We assume there

\footnotetext{
${ }^{1}$ The irradiance proxy could be from any number of sources.
} 


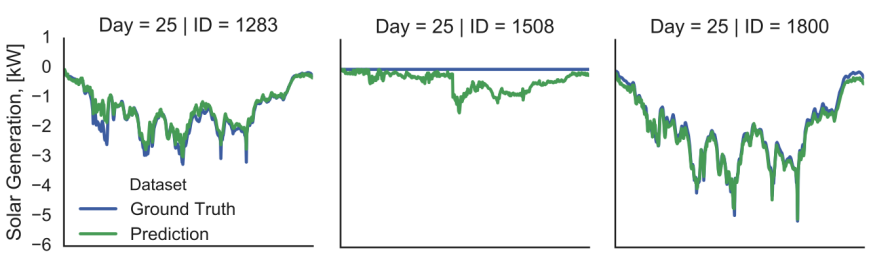

Figure 2: Solar disaggregation results

are $N$ households with solar generation and we define the customer load at each household $i$ time $t$ as $L_{t}^{i}$. We model the solar generation $S_{t}^{i}$ using the irradiance proxy measurement $\phi_{t}$. Furthermore, we assume we have individual AMI measurement at the customer level such that

$$
L_{t}^{i}+S_{t}^{t}=A M I_{t}^{i}
$$

Next, we define the aggregate load at the substation as $A L_{t}$. For simplicity in notation, we assume there are also $N$ households with load (i.e. there is no house without PV generation) and model the aggregate load as a function of outside temperature $f\left(T_{t}\right)$. Specifically, we model the temperature effects on load as piecewise linear as shown in 7]. We use the complete set of measurements and can cast the following contextually supervised source separation problem:

$$
\begin{array}{ll}
\underset{A L_{t}, L_{t}^{i}, S_{t}^{i}, C_{e f f}^{i}, \Theta}{\operatorname{minimize}} & \left\{\alpha\left(A L_{t}-\Theta f\left(T_{t}\right)\right)^{2}+\beta \sum_{i}^{N}\left(S_{t}^{i}-C_{e f f}^{i} \phi_{t}\right)^{2}\right\} \\
\text { subject to } & L_{t}^{i}+S_{t}^{t}=A M I_{t}^{i} \\
& \sum_{i=1}^{N} L_{t}^{i}=A L_{t}
\end{array}
$$

For the purposes of this initial investigation we used 2 days' data from 10 houses monitored by Pecan Street Inc. 8]. Pecan street sub-meters PV generation and load within the homes at a rate of $1 \mathrm{~min}^{-1}$. All houses are located in Austin, TX, and Pecan Street also provides hourly weather data for the time period that the homes are monitored.

\section{RESULTS}

Figure 2 shows actual versus disaggregated PV energy production from three households in the dataset. We observe that the proposed method works well for the homes with solar but not for the home without (ID\# 1508), which estimates a small amount of PV generation when in fact there is none. In future work we suggest improving the methodology by adding an $\ell_{1}$ regularization term for all values of $C_{\text {eff }}$ which will induce sparsity.

The disaggregation method is also able to recover otherwise unknown dynamics in load, shown in Figure 3 Load exhibits erratic behavior in all homes: ID\# 1283 exhibits what looks like duty cycling, and ID \#1800 exhibits large variability in the first half of the day but not during the second half. In both cases, the disaggregation method is able to recover this variability though there are no regressors in the linear model that might capture it.

E.g., a monitored PV generation unit nearby, irradiance measurements from a weather station, or estimated irradiance from satellite observations

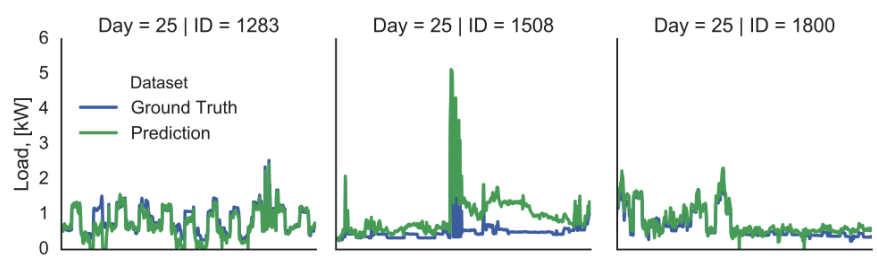

Figure 3: Load disaggregation results

\section{DISCUSSION}

In this paper, we disaggregate PV generation at individual households from historical AMI data. However AMI data are typically only available the day after collection, too late to be used in distribution system control. We plan to expand this work by conditioning our models on SCADA and weather data that are available in real time. We will then design the model to train on AMI data, but run in real-time to disaggregate $\mathrm{PV}$ generation and load from each home using only weather and SCADA data. This information is necessary for actively controlling distribution system equipment to prevent the adverse impacts of distributed PV, such as voltage violations and reverse power flow.

\section{REFERENCES}

[1] M. Ebad and W. M. Grady, "An approach for assessing high-penetration pv impact on distribution feeders," Electric Power Systems Research, vol. 133, pp. 347-354, 2016.

[2] M. Cohen and D. Callaway, "Effects of distributed pv generation on californiaâÁ Źs distribution system, part 1: Engineering simulations," Solar Energy, vol. 128, pp. 126-138, 2016.

[3] A. Nguyen, M. Velay, J. Schoene, V. Zheglov, B. Kurtz, K. Murray, B. Torre, and J. Kleissl, "High pv penetration impacts on five local distribution networks using high resolution solar resource assessment with sky imager and quasi-steady state distribution system simulations," Solar Energy, vol. 132, pp. 221-235, 2016.

[4] P. Gotseff, J. Cale, M. Baggu, D. Narang, and K. Carroll, "Accurate power prediction of spatially distributed pv systems using localized irradiance measurements," in PES General Meeting| Conference $\&$ Exposition, 2014 IEEE, pp. 1-5, IEEE, 2014.

[5] C. A. Gueymard, "Progress in direct irradiance modeling and validation," in Solar 2010 Conf., Phoenix, AZ, American Solar Energy Soc, 2010.

[6] E. C. Kara, C. M. Roberts, M. Tabone, L. Alvarez, D. S. Callaway, and E. Stewart, "Towards real-time estimation of solar generation from micro-synchrophasor measurements," arXiv preprint arXiv:160\%.02919, 2016.

[7] J. L. Mathieu, P. N. Price, S. Kiliccote, and M. A. Piette, "Quantifying changes in building electricity use, with application to demand response," IEEE Transactions on Smart Grid, vol. 2, no. 3, pp. 507-518, 2011.

[8] C. Holcomb, "Pecan street inc.: A test-bed for nilm," in International Workshop on Non-Intrusive Load Monitoring, Pittsburgh, PA, USA, 2012. 